\title{
On the integrability of stellar motion in an accelerated logarithmic potential
}

\author{
F. Namouni ${ }^{1}$, M. Guzzo ${ }^{2}$, and E. Lega ${ }^{1}$ \\ 1 Université de Nice, CNRS, Observatoire de la Côte d'Azur, BP 4229, 06304 Nice, France \\ e-mail: namouni@obs-nice.fr \\ 2 Dipartimento di Matematica Pura ed Applicata, Università degli Studi di Padova, via Trieste 63, 35121 Padova, Italy
}

Received 30 April 2008 / Accepted 21 July 2008

\section{ABSTRACT}

\begin{abstract}
Context. An accelerated logarithmic potential models the mean motion of stars in a flat rotation curve galaxy that sustains a wind system. For stars outside the galactic wind launching region, the asymmetric removal of linear momentum by the wind is seen as a perturbing acceleration superimposed on the galactic potential.

Aims. We study the integrability of stellar motion in an accelerated logarithmic potential.

Methods. We use surfaces of section of the dynamical system to probe the integrability of motion.

Results. We provide numerical evidence that motion in an accelerated logarithmic potential is non-integrable.

Conclusions. Large scale chaotic diffusion occurs for lower values of the projected angular momentum along the direction of acceleration and persists at all values of the angular momentum in the outer part of the galaxy inside the truncation radius where the galactic acceleration balances the wind-induced acceleration.
\end{abstract}

Key words. celestial mechanics - stars: kinematics - galaxies: kinematics and dynamics - galaxies: jets - chaos - diffusion

\section{Introduction}

New observations of galactic winds have shown that these processes are ubiquitous features in starburst and active galaxies (Veillet et al. 2005). In this paper, we develop the dynamical premise for studying the effect of asymmetric linear momentum removal from a galaxy with a flat rotation curve. Our investigation is motivated by the recent studies of the dynamical influence of asymmetric stellar jets on protoplanetary disks and the forming planets they contain (Namouni 2005, 2007; Namouni \& Zhou 2006; Namouni \& Guzzo 2007). These works have shown that the jet-induced acceleration leads to a number of dynamical consequences such as strongly modifying protoplanetary disk structure profiles, forcing the radial contraction and expansion of the disk, and enhancing the internal heating of the disk as it follows its varying state of least energy induced by the jets' variablity. Jet-induced acceleration also helps truncate the protoplanetary disk, excites the eccentricity of forming planets, and induces radial migration in binary systems. In view of the variety of these consequences, we expect that the wind-induced acceleration alters star orbits and affects the collective response of the galactic disk.

Modeling the motion of a star in a flat rotation curve galaxy as that of a test particle moving in a central logarithmic potential, $\log r$, where $r$ is the distance to the galactic center (Binney $\&$ Tremaine 1987), the asymmetric removal of linear momentum from the galaxy can in turn be modeled by an additional acceleration that does not depend on the position and velocity of the star. This approximation is valid as long as we consider the motion of stars outside the wind launching region. In effect, a star is affected only by the variation of mass and momentum losses inside its orbit because it revolves around the matter inside its orbit. It is important to note that the effect under consideration is not that of the direct action of the galactic wind on the star, such as that of the wind blowing on the star, which is physically negligible. Rather it is the indirect effect of the loss of linear momentum by the galactic disk on the motion of stars outside the wind launching region.

To set up the dynamical premise of this process, we first discuss the zero-velocity curves of the accelerated logarithmic potential as well as its orbits of least energy (Sect. 2). The surfaces of section allow us to show that orbital motion in the accelerated logarithmic potential is not integrable (Sect. 3), in contrast to the accelerated Kepler potential (Epstein 1916; Sommerfeld 1929; Landau \& Lifschitz 1969) that models orbital motion in the presence of a stellar jet (Namouni \& Guzzo 2007). Section 4 contains concluding remarks.

\section{Zero velocity curves and least energy orbits}

Galaxies with asymmetric wind systems lose linear momentum in the region inside the wind launching radius. The net momentum loss accelerates the wind launching region with respect to the matter outside it in the direction opposite to momentum loss (Namouni 2005, 2007; Namouni \& Guzzo 2007). In essence, asymmetric momentum loss from a wind system accelerates the inner part of the galaxy much like a rocket with respect to its outer part. Modeling the gravity of a flat rotation curve galaxy as a logarithmic potential (Binney \& Tremaine 1987), the motion of a star outside the wind launching region follows the equation:

$\frac{\mathrm{d}^{2} \boldsymbol{x}}{\mathrm{d} t^{2}}=-\frac{v_{\mathrm{c}}^{2}}{|\boldsymbol{x}|^{2}} \boldsymbol{x}+\boldsymbol{A}$,

where $\boldsymbol{x}$ denotes the position of the star in the galaxy, $v_{\mathrm{c}}$ is the galactic circular velocity and $\boldsymbol{A}$ is the wind-induced acceleration. 


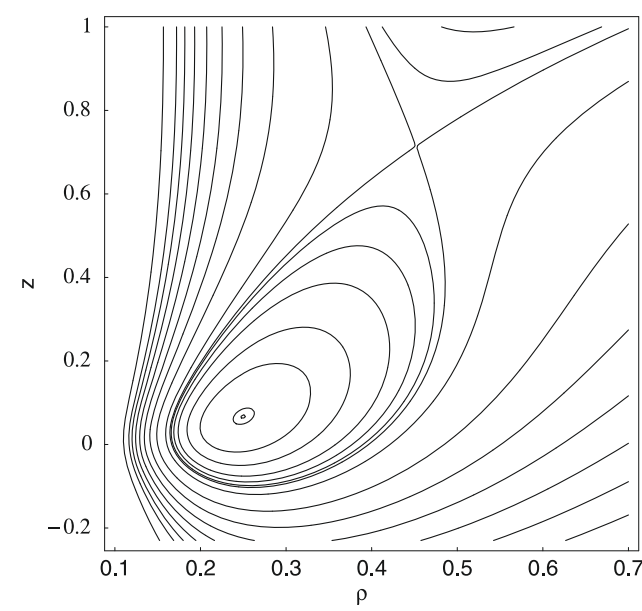

Fig. 1. Zero velocity curves for the value of $h_{z}^{2}=0.058313294$.

We examine the effect of momentum loss during a stationary wind episode and therefore take $\boldsymbol{A}$ to be constant in time. The presence of the acceleration $\boldsymbol{A}$ introduces natural units for time and distance. These are given as:

$L=\frac{v_{\mathrm{c}}^{2}}{A}$ and $T=\frac{v_{\mathrm{c}}}{A}$.

The former is related to the boundary of the logarithmic potential set by the equality of the gravitational acceleration and the windinduced acceleration. The latter is the typical excitation time for stellar orbits in the presence of acceleration. In terms of these units, the equations of motion are given as:

$\frac{\mathrm{d}^{2} \boldsymbol{x}}{\mathrm{d} t^{2}}=-\frac{\boldsymbol{x}}{|\boldsymbol{x}|^{2}}+\boldsymbol{e}_{z}$

where $\boldsymbol{e}_{z}$ is the unit vector along the direction of acceleration $\boldsymbol{A}$ that we choose as the $z$-axis of the inertial reference frame. Note that it is still possible to keep track of the relative strengths of the logarithmic potential and the constant acceleration. For instance the logarithmic potential is dominant for $|x| \ll 1$, and a unit orbital period corresponds to motion near the boundary where the gravitational acceleration balances $\boldsymbol{A}$.

As the acceleration is constant, the corresponding force derives from the potential $R=z$ and hence the dynamical system (3) admits the constant of motion:

$E=\frac{1}{2}\left(\dot{\rho}^{2}+\rho^{2} \dot{\theta}^{2}+\dot{z}^{2}\right)+\frac{1}{2} \log \left(\rho^{2}+z^{2}\right)-z$,

where $\rho, \theta$ and $z$ are the usual cylindrical coordinates. In addition to the energy $E$, the projection of the angular momentum $\boldsymbol{h}=\boldsymbol{x} \times \boldsymbol{v}$ along the direction of acceleration (or equivalently the $z$-axis), $h_{z}=\boldsymbol{h} \cdot \boldsymbol{e}_{z}=\rho^{2} \dot{\theta}$, is a constant of motion. Substituting the expression of $\dot{\theta}$ in terms of $h_{z}$ into Eq. (4), the energy equation now reads:

$E=\frac{1}{2}\left(\dot{\rho}^{2}+\dot{z}^{2}\right)+\frac{h_{z}^{2}}{2 \rho^{2}}+\frac{1}{2} \log \left(\rho^{2}+z^{2}\right)-z$.

The zero velocity curves of the system (3) are obtained from the effective potential of the energy equation - the last three terms of (5). In Fig. 1, we show such curves for the value of $h_{z}^{2}=0.058313294$. The region of bounded motion is restricted in space inside a radius of $\rho^{2}+z^{2} \simeq 1$ where the gravitational attraction exceeds the amplitude of the perturbing acceleration. There

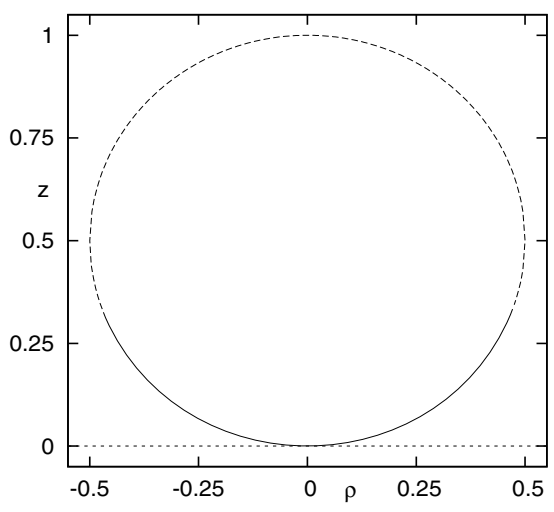

Fig. 2. Sphere of least energy orbits. The solid (dashed) line denotes stable (unstable) orbits.

are two equilibrium (least energy) points located at $(\rho=0.25$, $z=0.067)$ and $(\rho=0.452, z=0.714)$. The former is a stable orbit and the latter is unstable.

We determine the properties of least energy orbits by equating with zero the partial derivatives of $E$ with respect to $\rho, z, \dot{\rho}$ and $\dot{z}$. The derivatives with respect to $\dot{\rho}$ and $\dot{z}$ show that least energy orbits are circular and planar. The derivative with respect to $\rho$ gives the expression of $h_{z}$ :

$h_{z}^{2}=\frac{\rho^{4}}{\rho^{2}+z^{2}}$

or equivalently the orbital rotation rate $\Omega=\dot{\theta}=h_{z} \rho^{-2}$ in terms of $\rho$ and $z$. Lastly, the derivative with respect to $z$, which is the vertical projection of the equation of motion, shows that least energy orbits hover above the center of gravitational attraction according to the equation:

$z=\frac{1 \pm \sqrt{1-4 \rho^{2}}}{2}$

From this equation, it is seen that the locus of least energy circular orbits is a sphere centered on $(\rho=0, z=1 / 2)$ of radius $1 / 2$ (Fig. 2). The upper and lower signs do not correspond to the stability of the equilibrium orbits. To determine which orbits are stable on the least energy sphere, we first note that each least energy orbit shown in Fig. 2 has a different value of the projected angular momentum, $h_{z}$, and the energy, $E$. From the zero velocity curves (Fig. 1), we know that for a given value of $h_{z}$ there are two least energy orbits, one stable and one unstable the smallest value of $h_{z}$, zero, must corresponds to the two least energy points at $\rho=0$ and $z=0$ (stable) and $z=1$ (unstable). Using Eq. (7), it is possible to express $h_{z}(6)$ as a function of $z$ for least energy orbits and solve for the values of $z$ (and hence $\rho$ ) that determine the stable and unstable orbits. This substitution yields:

$h_{z}^{2}=z(z-1)^{2}$.

This shows that $h_{z}^{2}$ has a maximum at:

$z_{\mathrm{b}}=1 / 3$, and $\rho_{\mathrm{b}}=\sqrt{2} / 3$,

and as expected $h_{z}$ vanishes for $z=0$ and $z=1(\rho=0)$. The maximum value of $h_{z}^{2}$ is $4 / 27$ and corresponds to the boundary of stable least energy orbits. It also defines the truncation radius of the galaxy. The condition

$\left|h_{z}\right|<2 / 3 \sqrt{3}$ 

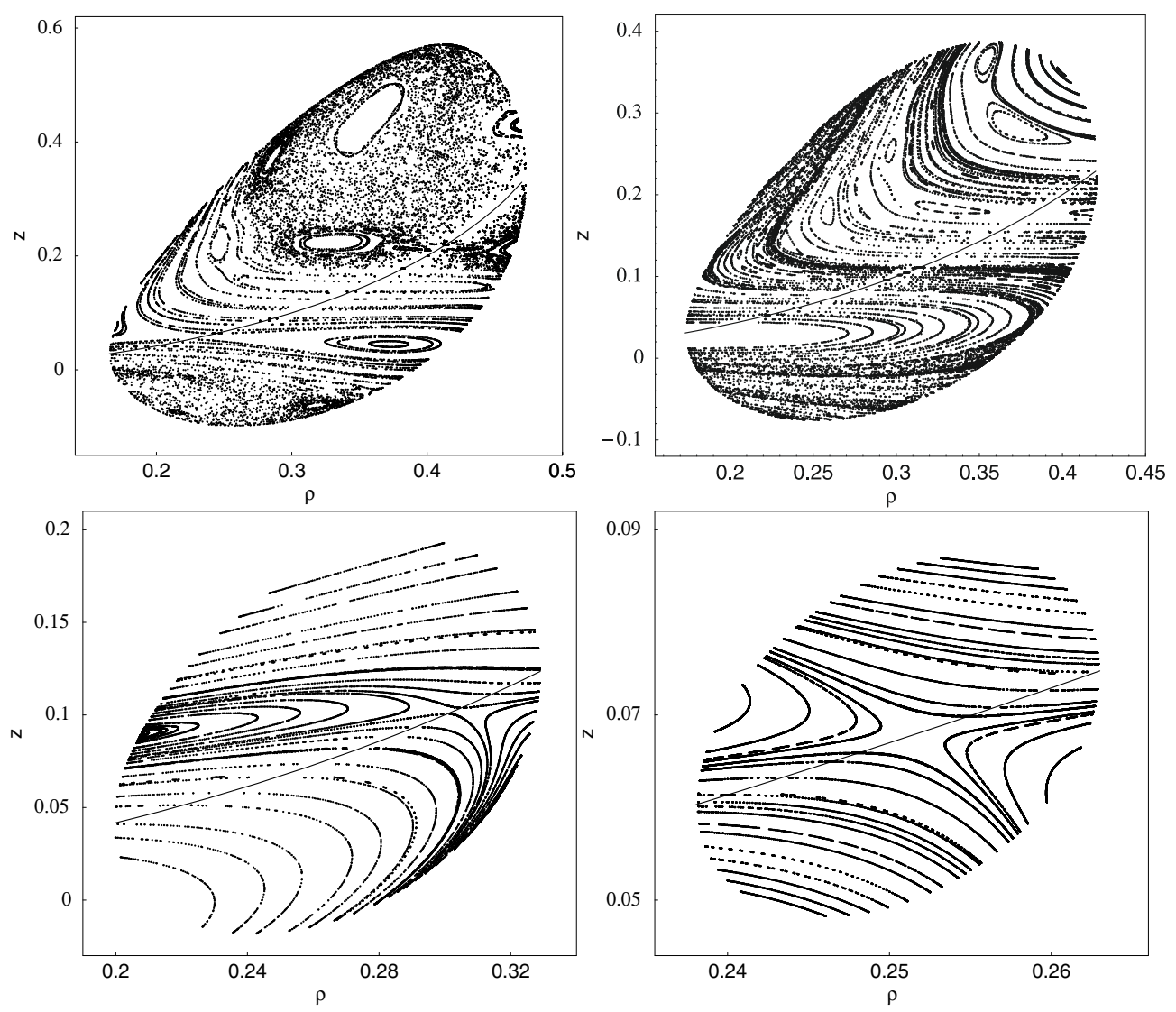

Fig. 3. Surfaces of section for $h_{z}^{2}=0.058313294$. The energy levels are $E=-0.75$ (top left), $E=-0.80$ (top right), $E=-0.90$ (bottom left) and $E=-0.95$ (bottom right). The solid line is the sombrero profile $z=\left(1-\sqrt{1-4 \rho^{2}}\right) / 2$ that divides space into $\ddot{z}<0$ orbits (above the line) and $\ddot{z}>0$ orbits (below the line).

is therefore necessary for bounded motion to occur. The profile of stable and unstable orbits is shown in Fig. 2 with solid and dashed lines respectively. The locus of the stable orbits in the $\rho z$-plane has a profile similar to (but less flat at its base than) the sombrero-shaped profile associated with the accelerated Keplerian potential (Namouni 2007; Namouni \& Guzzo 2007). For consistency with the Keplerian case, we call the locus of the stable orbits of an accelerated galactic potential the sombrero profile. An additional difference between the accelerated Keplerian and logarithmic potentials is that the former admits a global third integral of motion implying that orbits are everywhere regular - even in the vicinity of the zero-velocity separatrix and the unstable least energy orbit. The accelerated logarithmic potential does not have a global third integral. This we show in the next section.

\section{Surfaces of section}

The integrability of stellar motion in the accelerated logarithmic potential may be determined numerically by drawing surfaces of section of the dynamical system (3). Sections in phase space are chosen for (i) a constant energy, $E$; (ii) a constant projected angular momentum, $h_{z}$; and (iii) with the condition $\dot{z}=0$. Sections usually require a crossing direction such as that given by $\ddot{z}>0$. However, as we find it convenient to represent sections in the $\rho z$ plane, we can readily determine the sign of $\ddot{z}$ everywhere in that plane. As $\ddot{z}$ equals the vertical component of the gravitational potential augmented by the perturbing acceleration, $\ddot{z}$ vanishes exactly on the sphere of least energy (Eq. (7)). The sign of $\ddot{z}$ is negative inside the least energy sphere and positive outside it. This allows us to explore numerically the whole area inside the zero velocity curve associated with the values of $E$ and $h_{z}$, and know the sign of $\ddot{z}$. Accordingly, we plot the curve of $\ddot{z}=0$ (Eq. (7)) on all surfaces of section which are now allowed to include both $\ddot{z}>0$ orbits and $\ddot{z}<0$ orbits.

Using the same value of the sombrero orbit as that of Fig. 1, $\rho=0.25$, we set $h_{z}^{2}=0.058313294$ and compute the surfaces of section for $E=-0.95,-0.90,-0.80$ and -0.75 . The value $E=$ -0.95 corresponds to a small region around the sombrero orbit. Increasing the value of $E$ widens the area of possible motion. Orbits have been computed for a duration $t=200$ as well as for various values of $\rho, z$ and $\dot{z}$ whereas $\dot{\rho}$ is obtained from the energy equation.

The section for $E=-0.75$ (Fig. 3, top left) shows that motion in the accelerated logarithmic potential is not integrable with large chaotic regions developing for smaller and larger elevations $z$. Irregular motion is milder near the plane $z=0$ that contains the gravitational center. Noting that the surface of section is asymmetric with respect to the sombrero profile $z=\left(1-\sqrt{1-4 \rho^{2}}\right) / 2$, restricting the surface of section to positive $\ddot{z}$ only would have lead to a loss of information on the motion inside the least energy sphere. The lowering of the value of $E$ shrinks down the region of possible motion and as well as that of irregular motion (Fig. 3, top right). In particular for $E=-0.90$ motion is further restricted to a small region around the sombrero orbit $(\rho=0.25, z=0.067)$ and an unstable point 

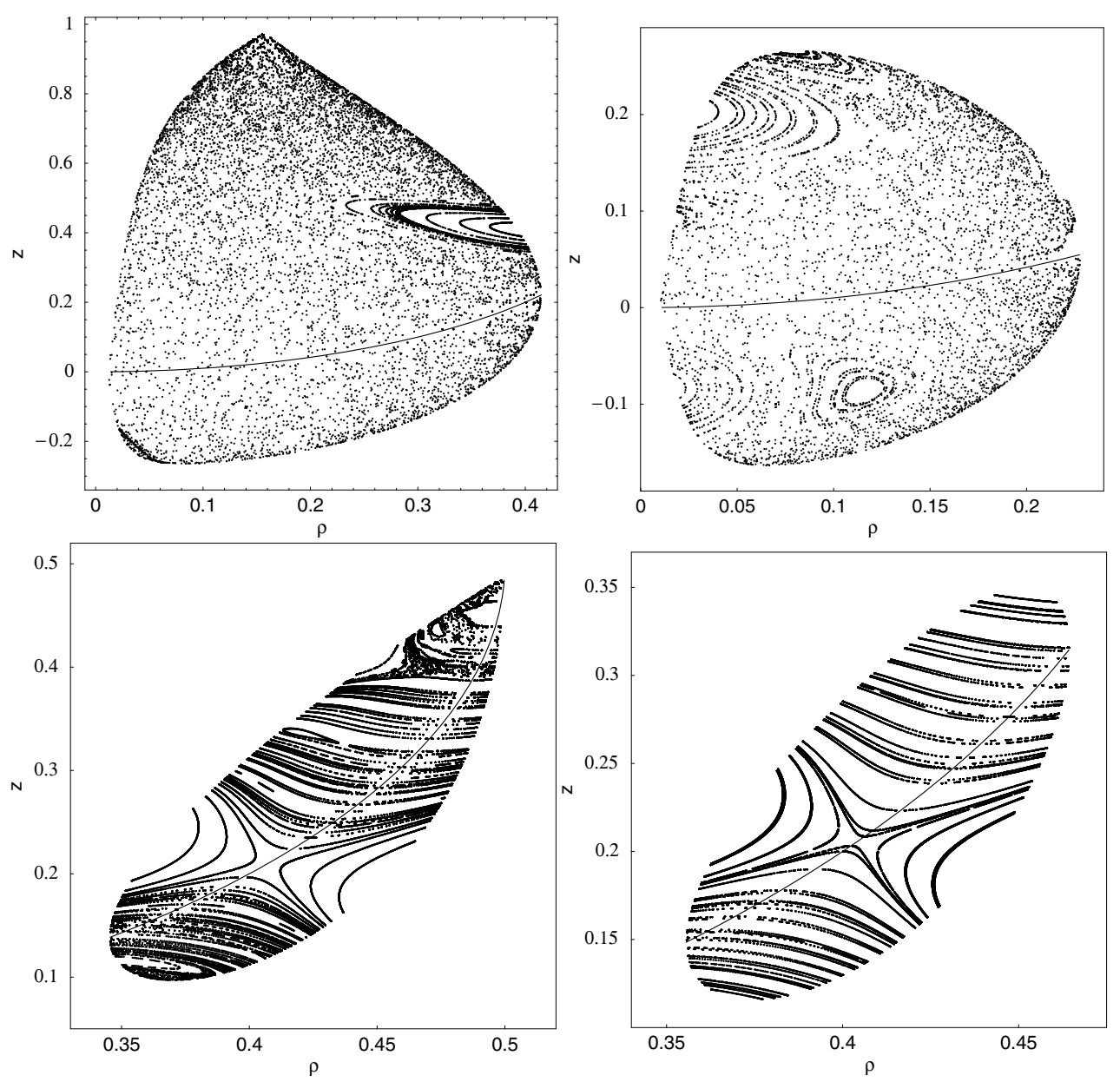

Fig. 4. Surfaces of section for different values $h_{z}^{2}=6.24 \times 10^{-4}$ (top row) and $h_{z}^{2}=0.128$ (bottom row). For $h_{z}^{2}=6.24 \times 10^{-4}$, the energy levels are $E=-0.97485$ (top left) and $E=-1.5$ (top right). For $h_{z}^{2}=0.128$, the energy levels are $E=-0.590575$ (bottom left) and $E=-0.596$ (bottom right). The solid line is the sombrero profile $z=\left(1-\sqrt{1-4 \rho^{2}}\right) / 2$.

appears very near it (Fig. 3, bottom panels). Lowering the energy further makes the unstable point converge towards the sombrero orbit ( $E=-0.95$, Fig. 3, bottom right) implying the presence of a mild separatrix-type diffusion in the vicinity of the least energy orbit.

The extent of the regions of chaotic motion also depends on the value of the angular momentum $h_{z}$. We find that the smaller the projected angular momentum $h_{z}$ the larger the chaotic motion regions. This is shown in Fig. 4 where we plot surfaces of section for $h_{z}^{2}=6.24 \times 10^{-4}$ and 0.128 . The former corresponds to a stable sombrero orbit at $\rho=0.025$ and $z=6.25 \times 10^{-4}$ and the latter to $\rho=0.4$ and $z=0.2$. In Fig. 2, these two orbits are on each side of that corresponding to Fig. 3. For $h_{z}^{2}=6.24 \times 10^{-4}$, the energy levels are $E=-0.97485$ (top left) and $E=-1.5$ (top right). The former has been chosen close to the energy value of the unstable least energy orbit. The region of chaotic motion is large and remains so even when the energy value is lowered ( $E=-1.5)$ so that the zero velocity curve extent (i.e. the size of the surface of section) is 4 times smaller in the vertical direction that it is for $E=-0.97485$. For $h_{z}^{2}=0.128$, the energy levels are $E=-0.590575$ (bottom left) and $E=-0.596$ (bottom right). As for the previous value of $h_{z}$, the former energy has been chosen close to the value of the unstable least energy orbit. This time, the region of chaotic motion is confined to the vicinity of the unstable orbit and disappears when the energy value is lowered slightly $(E=-0.596)$ so that the zero velocity curve extent is only 1.4 times smaller in the vertical direction that it is for $E=$ -0.590575 . We conclude that for the smaller $h_{z}$, the extent of the chaotic region is much larger than that of Fig. 3 and irregular motion dominates the surface of section even when the energy value is far from that of the unstable least energy orbit. For the larger value of $h_{z}$, extended chaotic motion is confined to the vicinity of the unstable least energy orbit as soon as the energy level is lowered away from that of the unstable orbit, and no region inside the zero velocity curve shows large scale chaotic motion.

\section{Concluding remarks}

In this paper we showed that stellar motion in an accelerated logarithmic potential is not integrable unlike motion in an accelerated Keplerian potential. We have used zero-velocity curves, least energy orbits as well as surfaces of section to determine the global topology of orbital motion. We find that for a given projected angular momentum, the larger the area of the zerovelocity curve in $\rho z$-space, the larger the fractional size of the chaotic motion region. The size of the chaotic motion regions also depends on the value of the projected angular momentum $h_{z}$. The smaller $h_{z}$, the larger the extent the chaotic motion region. Smaller values of $h_{z}$ correspond to inner sombrero orbits (i.e. $\rho \ll 1)$ while larger values of $h_{z}$ correspond to zero velocity curves whose corresponding motion is confined near to the 
truncation radius of the galaxy (i.e. the stable and unstable least energy orbits become close).

For the diffusion that we have uncovered in this work to affect stellar orbits, the local diffusion time has to be shorter than the age of the galaxy. This implies that in the outer regions where the dynamical time (i.e. the orbital period $2 \pi r / v_{\mathrm{c}}$ ) is comparable to the age of the galaxy, diffusion does not have enough time to occur. The reason is that the local diffusion time is always and everywhere larger than the local dynamical time. For instance, the scatter of points in the top left panel of Fig. 3 has been obtained after 200 time units - a time unit being the dynamical time at the truncation radius of the galaxy, $T$ (2). For a typical galaxy, such a timespan is greater than the age of the Universe.

Diffusion therefore affects stellar orbits in an accelerated flat rotation curve galaxy for low values of the projected angular momentum $h_{z}$ and well inside the truncation radius (i.e. the outer edge of the sombrero profile). Stellar orbits near sombrero orbits are more regular as diffusion is negligible. In these parts of the galaxy, it is possible to make use of secular perturbation techniques to ascertain the evolution of stellar orbits whose periods are small compared to the duration of the wind episode.

Acknowledgements. The authors thank the referee for useful comments.

\section{References}

Binney, J., \& Tremaine, S. 1987, Galactic Dynamics (Princeton: Princeton University Press)

Epstein, P. S. 1916, Ann. der Phys., 50, 489

Landau, L. D., \& Lifshitz, E. M. 1969, Course of Theoretical Physics (Oxford: Pergamon Press)

Namouni, F. 2005, AJ, 130, 280

Namouni, F. 2007, ApJ, 659, 1505

Namouni, F., \& Zhou, J. L. 2006, CMDA, 95, 245

Namouni, F., \& Guzzo, M. 2007, CMDA, 99, 31

Sommerfeld, A. 1929, Atombau und Spektrallinen (Braunschweig: Vieweg, Sohn \& Braunschweig)

Veilleux, S., Cecil, G., \& Bland-Hawthorn, J. 2005, ARA\&A, 43, 769 